\title{
Issues in Identification and Assessment of Children with Autism and a Proposed Resource Toolkit for Speech-Language Pathologists
}

\author{
Yvette Hus \\ TAV College, Montreal, QC, Canada
}

\section{Keywords}

Autism issues - Speech-language pathologists · Resource toolkit

\begin{abstract}
Background: The prevalence of autism spectrum disorder (ASD) has increased significantly in the last decade as have treatment choices. Nonetheless, the vastly diverse autism topic includes issues related to naming, description, identification, assessment, and differentiation from other neurodevelopmental conditions. ASD issues directly impact speech-language pathologists (SLPs) who often see these children as the second contact, after pediatric medical practitioners. Because of shared symptomology, differentiation among neurodevelopmental disorders is crucial as it impacts treatment, educational choices, and the performance trajectory of affected children. Objectives: To highlight issues in: identification and differentiation of ASD from other communication and language challenges, the prevalence differences between ASD gender phenotypes, and the insufficient consideration of cultural factors in evaluating ASD in children. A second objective was to propose a tool to assist SLPs in the management of autism in children. Summary: A
\end{abstract}

\section{KARGER}

(c) 2017 S. Karger AG, Basel

E-Mail karger@karger.com

www.karger.com/fpl universal resource toolkit development project for SLP communities at large is proposed. The resource is comprised of research-based observation and screening tools for caregivers and educators, as well as parent questionnaires for portraying the children's function in the family, cultural community, and educational setting.

(c) 2017 S. Karger AG, Basel

\section{Introduction}

Autism, a complex and chronic neurodevelopmental condition, is to a great extent the children's failure or difficulty in acquiring social interaction and communication, and language to support these abilities [1]. These children inevitably require speech-language pathologists' (SLPs) interventions. SLPs are not routinely included in assessments of children with autism spectrum disorder (ASD) [2] either because of insufficient training in using the recommended standard instruments or because of gaps in regional or local governmental policies regarding who should be diagnosing ASD and what criteria or tools to use [3]. Nevertheless, SLPs' awareness and understanding of issues surrounding aspects of ASD differentiation, 
identification, and assessment are critical for intervening effectively with these children and for serving as a vital resource to their families. Recently, the American Academy of Child and Adolescent Psychiatry Committee on Quality Practice [4] recommended a multidisciplinary team approach in ASD evaluation. If SLPs are to participate successfully in such teams, knowledge of the issues in which the autism topic is immersed is crucial. This article has two aims. First, to highlight issues and gaps surrounding $\mathrm{ASD}$, a topic that has generated much research and discussion in the past decades [1]. Second, to encourage and empower SLPs in their practice with children and adolescents with ASD by proposing the development of a universal and culture-specific resource toolkit modelled on the ASD pediatric management guide by Johnson and Myers [5]. The theoretical bases proposed for the toolkit are: (1) transcultural principles of psychiatry [6,7], a multidisciplinary field which acknowledges that culture and ethnic identity are embedded in the awareness and interpretation of disease so that these must be taken into account when examining individuals, and (2) a bioecological model [8,9], where family factors, services, school, community, and various societal resources are considered since they play a vital role in determining the developmental outcomes of children with ASD [10,11].

\section{Issues and Challenges in Diagnostics}

\section{The Problem of Heterogeneity of Symptoms}

Although ASD is classified as a heritable biological condition, there is not yet a medical or genetic test to verify its existence as for fragile X syndrome (FXS), Williams syndrome, and Down syndrome $[12,13]$. These syndromes, marked with intellectual and language challenges, may occur with ASD, particularly in individuals with FXS [14]. Their ASD diagnosis, however, is based on behavioral observations and reports $[15,16]$. Depending on cognitive function and assessment age, children with ASD demonstrate widely varied phenotypes, and wideranging symptom severity and developmental paths, making accurate and stable ASD identification a challenging process, especially in early development [11].

Temple Grandin, a high functioning person with autism, provides poignant insights into the extent of variability in symptom manifestations she suffered in early childhood. Her speech was significantly delayed, and her behavior was seriously dysregulated. With SLP intervention, she started speaking at about age 3.6, and her behavior was "normalized" until she became tired, when
“... bouts of impulsive behavior would return." [17, p. 144]. She highlights the individual differences in response to sensory stimuli amongst those on the spectrum. For example, she may love the very same visual stimuli that cause others with autism "great pain, fear, and discomfort." Similarly, she notes, some children may enjoy the sound of running water while others "run away in terror" [18].

Researchers identified other factors impeding accurate ASD identification, especially in very young children. Charman [19] notes that although desirable, reliable early identification is hampered due to regression or loss of skills; by age 2 they may exhibit reduced orienting to name, poorer joint attention, some early motor abnormalities, and reduced emotional expression. Meilleur and Fombonne [20], for example, identified a subgroup of children on the autism spectrum with communication and language loss that occurred between ages 15 and 24 months. The researchers $[19,20]$ note that this regression is rare in other non-ASD early developmental disorders. Recently, however, a longitudinal study of ASD developmental trajectories revealed a toddler-age subgroup of non-ASD siblings with noted declines in cognitive and linguistic skills, underscoring the importance of surveilling the development of these high-risk groups [21].

Another well-known difficulty is the marked heterogeneity in cognitive, communication, and linguistic abilities of children with ASD [22-26]. Language skills span the entire continuum from no speech or functional speech, to mastery levels of comprehension, production, and literacy, while autism severity ranges from very severe to very mild. The greatest variability, however, occurs in their intellectual abilities (IQ) and cognitive profiles (verbal vs. nonverbal IQ) [27, 28]. Although IQ appears as a strong predictor of social and adaptive functioning in young children, adaptive behavior was determined to be most dysfunctional in older children, despite IQ [23], indicating the wisdom in employing ongoing surveillance with these children.

\section{Identification and Differentiation Issues}

According to the Unites States Environmental Protection Agency [29], attention deficit hyperactivity disorder (ADHD), dyslexia, developmental coordination disorder (dyspraxia), specific language impairment (SLI), and ASD are amongst the most common childhood neurodevelopmental disorders affecting about $15 \%$ of children aged 3-17 years. These present a challenge in accurate differentiation because all have complex and multifactorial etiologies including high heritability, and symptoms 
that vary with age and between individuals. Further hampering accurate identification is the high rate of comorbidity or shared symptomology, which as Gillberg [30] notes is "the rule rather than the exception in child psychiatry and developmental medicine." The twin studies of Lichtenstein et al. [31] showed common genetic underpinnings between ASD and ADHD, developmental coordination disorder, tic disorder, and learning disabilities, thus providing an explanation for the high comorbidity between these disorders. It further highlights the complexity and difficulty in accurate ASD diagnosis.

Delayed language development is a hallmark in young children with ASD, making it difficult to differentiate the type of language delay characteristic of children with ASD from non-ASD delayed language development [32, 33]. Studies of 2- to 3-year-olds with ASD and a non-ASD delayed language development group matched on expressive language confirmed their communication and language delay when compared to typical toddlers, but they were strikingly similar to each other in communication, social interaction, and speech development delays. Those with ASD, however, were more delayed in nonverbal cognitive skills, visual perception and fine motor development, social and communicative language, including gestures and pretend play.

The ASD phenotype also overlaps with SLI [1, 34-36]. Both are heritable and share deficits in social and communicative development, syntax, and lexicon [37, 38], giving the impression of comorbidity. Despite their prominent language difficulties, comorbidity was not confirmed by their genetics $[34,35,39,40]$. Bishop [35, p. 624 ] employs the concept of phenomimicry to explain that the ASD phenotype can lead to language impairment but the resemblance to SLI is superficial as the SLI genetic risk factors are absent in those with ASD and language impairment. In fact, Tager-Flusberg [41] highlights the risk factors shared by ASD and SLI, such as family history and being a boy, limited gestural communication, poor receptive language, delays in motor development, and atypical neural responses to auditory stimuli. However, uncovering the risk factors associated specifically with SLI is hampered due to the imbalance between the larger amount of ASD research compared to that in SLI, fewer SLI developmental studies, and the variability in research methodologies and paradigms.

\section{Identification of ASD in FXS}

FXS is the most frequent cause for intellectual disability, and a significant risk for ASD, rendering it the most common genetic disorder that is comorbid with ASD
[12]. About $25 \%$ of individuals with FXS meet the criteria of ASD comorbidity [14]. The FXS diagnosis, though, is based on DNA tests while at present none exist for ASD, making it difficult to identify ASD in FXS. Nonetheless, as Klusek et al. [42] note, identifying FXS with ASD is critical since those with the comorbidity are significantly more impaired on cognitive and expressive language, have greater behavior issues, show greater social impairment, and poorer adaptive functions. They are also at increased risk of developing medical issues. Affected children are not likely to overcome their autistic behaviors without specialized intervention. ASD comorbidity, therefore, should be a key factor in determining type and intensity of the interventions they require.

\section{Issues in Identification of Girls with ASD and an}

\section{Emerging Female Phenotype}

Researchers ascertained that ASD occurs more often in boys than girls, with ratios ranging from $2: 1$ to $5: 1$ [4346]. Gender/sex differences are not intrinsic to all neurodevelopmental disorders. For example, gender differences are not attributed to dyslexia [47] but found in ADHD, affecting more boys than girls, with or without ASD [48]. Clearly, gender differences in ASD with ADHD reflect those found in typical populations [49]. The causes underlying gender differences are not clear. Baron-Cohen [43] proposes "an extreme male brain" theory, whereby inherent gender brain differences underlie the more pronounced ASD symptoms in males more so than in females. He proposed two factors to clarify this discrepancy: (1) a female empathizing factor and (2) a male systematizing factor. Evidence for the hypothesized brain profiles underlying gender differences came from studies of typical children, boys and girls with ASD [50], and studies of typical and high functioning adults with ASD from diverse geographical regions [51].

While no gender-specific ASD phenotype was established in toddlers $[44,52]$, a noted difference not reflected in the gender identification ratio was found in young children $[44,53,54]$. The IQ factor and test instrument limitations are used to explain the discrepancy [55-57]. Girls with ASD and concomitant low IQ show symptoms that are often more severe than those in boys and so meet ASD criteria easily. Those with average IQ have better social behavior and more subtle autism symptoms, making it more difficult to meet the cutoff scores on diagnostic tests so that girls with ASD may go undetected. Similarly, standard test instruments designed around male ASD symptoms may underlie the higher prevalence of FXS boys with ASD than of girls [41]. 
Table 1. Qualitative characteristics of verbal girls with ASD without cognitive impairment when compared to boys as described by Lai et al. [46]

\begin{tabular}{|c|c|}
\hline Domain & Behaviors \\
\hline Social interaction & $\begin{array}{l}\text { Show greater awareness of the need and desire for social interaction } \\
\text { Tend to behave in a "shy" manner } \\
\text { Copy or imitate others and mask their problems by developing compensatory } \\
\text { strategies } \\
\text { Have only one or few close friends } \\
\text { Tend to be "mothered" by their peers in primary school } \\
\text { Often bullied in high school }\end{array}$ \\
\hline Communication & $\begin{array}{l}\text { Have better linguistic abilities } \\
\text { Have better imagination and pretend play } \\
\text { Pretend play mostly nonreciprocal and overly structured }\end{array}$ \\
\hline $\begin{array}{l}\text { Restricted repetitive patterns } \\
\text { of behavior, interests, or } \\
\text { activities }\end{array}$ & $\begin{array}{l}\text { Their interests involve people and animals rather than objects and things } \\
\text { Topics and activities more typical of non-ASD girls (often missed as an ASD } \\
\text { symptom) }\end{array}$ \\
\hline Personality characteristics & $\begin{array}{l}\text { Show a tendency to be perfectionist and determined } \\
\text { Tend to be controlling in play with peers } \\
\text { Show oppositional stance } \\
\text { Periodically exhibit eating problems }\end{array}$ \\
\hline
\end{tabular}

\section{An ASD Female Phenotype Emerges}

While some researchers found no differences between nonverbal boys and girls with ASD in terms of cognitive and adaptive skills [45], discrepant results were found for verbal children with ASD. These researchers note the difficulties inherent in IQ measurements of ASD children and in matching IQs for data analyses (e.g., some IQ results were based on a receptive vocabulary test). In addition, they found that in some ASD databases, verbal girls with ASD showed lower cognitive and social abilities than boys, while in others verbal girls with ASD had similar or better skills than boys. The discrepancies in findings make it difficult to determine gender phenotypes in ASD. However, Lai et al. [46] note that although standard test instruments may not provide numeric criteria for identifying girls, qualitative differences between boys and girls with ASD without intellectual disability describe a female ASD phenotype. Table 1 delineates the skill domains and the qualitative differences between genders. The noted developmental differences between genders may reflect biological underpinnings and cultural expectations of girls. Notwithstanding gender differences, accurate diagnosis of ASD in both genders is essential so that affected individuals can receive the support and resources for effective development [44]. Furthermore, in preparation for their futures, allocated human and monetary resourc- es should center on job training and employment to ensure independence and a sustaining quality of life.

\section{Issues in Current ASD Classification}

Despite the heterogeneity of ASD manifestations in affected children, they share core deficits delineated in the DSM-5 of the American Psychiatric Association [22]. It includes descriptors of ASD symptoms with severity level criteria and symptoms of social (pragmatic) communication disorder (SPCD) although without assigned severity levels. A diagnosis of ASD requires evidence of impairment in three features of social communication and social interaction and two features of restricted repetitive patterns of behavior (RRPB). Symptoms must be present in early development and cause impaired everyday function. Both social communication and social interaction, and RRPB severity levels are based on the degree of support the individual needs in order to function: mild, substantial, and very substantial support. The diagnosis should also specify presence or absence of accompanying intellectual impairment, language impairment, or whether there are associated known medical, genetic, or other conditions.

SPCD includes difficulties in social verbal and nonverbal communication. However, this presents a problem since it requires sufficiently developed language levels to 
detect symptoms in pragmatic language. Hence, deficits may only be apparent after age 4 , while mild deficits may only be evident in early adolescence [58]. The SPCD diagnosis hinges on evidence of impairment regarding four features but absence of RRPB. Differentiating ASD from SPCD presents a specific challenge as the deficits detailed in SPCD overlap sufficiently with the social communication impairments defined in ASD to cause confusion [58]. Rosin [58] advises that those who do not exhibit at least two features in RRPB be diagnosed with SPCD. However, RRBPs may have been present in the child's developmental history but were resolved, and went unnoticed or unreported by their caregivers $[59,60]$, potentially compromising accurate differentiation at the time of diagnosis. Norbury [61] questions the utility of treating SPCD as a separate category as its symptoms are shared by other neurodevelopmental and genetic disorders, and its diagnosis is hampered by a lack of reliable and culturally valid assessment measures. Norbury suggests that it best be treated as a dimensional symptom profile that is typically shared by various disorders.

Brukner-Wertman et al. [62] highlight difficulties arising from the DSM-5 ASD classification as it imposes categories on a spectrum disorder with symptom continuums. Furthermore, considering ASD and SPCD as separate categories cannot be substantiated as detection of ecological pragmatic impairment is actualized in research mostly with instruments that have not yet been validated for clinical use. They also question describing severity levels in ASD only, and lumping RRPB with social communication as it can lead to differentiation problems (e.g., RRPB also defines tic disorder).

Issues in Screening and Diagnostic Test Psychometrics

Successful screening or diagnostics reside in the psychometric strength of the instruments used $[63,64]$. Test sensitivity refers to how correctly tests identify those with the disease: true positive means individuals have the disorder and the test is positive; false negative means that the person has the disease but the test is negative (sensitivity $=$ true positives $\div$ true positives + false negatives), so that a test with $70 \%$ sensitivity misses $30 \%$ of those that actually have the disorder (false negatives). Specificity refers to how correctly tests identify those without the disorder: true negative indicates the person does not have the disease and the test is negative; false positive implies the person does not have the disorder but the test is positive (specificity $=$ true negatives $\div$ true negatives + false positives), e.g., $80 \%$ specificity correctly identifies those without the problem but misidentifies $20 \%$ as having it (false

Identification and Assessment of Children with Autism: A Proposed Resource Toolkit positive). Positive predictive value (PPV) and negative predictive value (NPV) refer to the likelihood of finding those with the disease (PPV $=$ true positive $\div$ true positives + false positives) and without the disease (NPV $=$ true negative $\div$ true negative + false negatives). These are used when considering the prevalence of the disease/disorder in a clinical population. PPV is expressed as percent probability so that the higher the PPV, the greater the diagnostic accuracy. Lalkhen and McCluskey [63] explain that PPVs are generally lower in universal screenings because of a greater number of false positives, and higher when drawn from a clinical population with a greater prevalence of the disorder.

Screening and diagnostic ASD instruments showing at least $80.8 \%$ sensitivity in correct identification are considered as gold standard. The Autism Diagnostic Interview-Revised (ADI-R) [65] and Autism Diagnostic Observation Schedule (ADOS-2) [66] are the current gold standard instruments recommended for accurate diagnosis. Brukner-Wertman et al. [62] point out difficulties with these instruments. The ADI-R represents a mismatch as it was designed with DSM-IV criteria and is used to determine DSM-5 symptoms. The instrument tends to miss mild ASD forms, while the ADOS-2 combines social communication and RRPB symptoms so that it does not distinguish SPCD from ASD. Researchers worry that the present DSM-5 two-category classification and diagnostic instrument weaknesses will contribute to SPCD misdiagnosis causing deprivation of treatment that is more often reserved for children with an ASD diagnosis [61, 62]. However, Falkmer et al. [67] showed they are evidence-based, with the highest sensitivity and specificity of the tests reviewed. When used in combination, their accuracy levels were very similar to the gold standard. For example, combined, they correctly identified ASD in a group of young children with developmental language delay [68]. Furthermore, ADOS with revised cutoff scores improved sensitivity so that it successfully identified mild ASD [69]. However, Lord [70] points out that although these are gold standard instruments, they were not validated on multicultural populations, and so may not adequately identify and diagnose culturally and linguistically diverse (CLD) children. In addition, Werling and Geschwind [54] suggest that the male bias in ASD identification points to a mismatch between the diagnostic criteria used by the instrument and the female ASD presentation in those with at least an average IQ. The lower ability of girls than boys with similar ASD symptom levels to meet diagnostic criteria was noted by other researchers [45]. 
Charman and Gotham [16] report that there are many measurement issues in screening and diagnostic instruments, with problems often arising when screening instruments are used for clinical diagnosis. Although there are a number of validated ASD screening tools [71], screening in general populations produces low PPVs. However, when screening tools are used for surveillance (i.e., when combined with parental voiced concerns, observations, and clinician judgement), their efficacy improves markedly. Based on reviews of screening studies, Charman and Gotham are opposed to ASD universal screening in children aged 5 years and younger because it increases costs, and the problem of overidentification with its corollary of causing anguish to parents of "failed" children. Nonetheless, researchers around the globe advocate for the detection of ASD between 9 and 24 months as it may lead to early treatment and improved outcomes for the affected child [72-76]. However, following an extensive review of screening accuracy, benefits, and potential harm of both early screening and early behavioral treatments, Siu [77] does not recommend early screening for children who have not been diagnosed with developmental delays and are not signaled by their caretakers.

\section{CLD Populations}

It is now evident that ASD exists in all racial, ethnic, and socioeconomic groups within Western, and nonWestern countries. Its core deficits are the same in all cultures, but its symptoms and developmental course are shaped by the cultural context in which the child lives [78-80]. Although autism exists around the globe and across numerous cultures, most of the research and assessment instruments come from Western cultures. This is problematic since awareness and concepts about "illness" vary between cultures, geographical regions, or even within regions $[6,7]$. An ASD survey in two USA states $[78,81]$ showed practitioners more often misidentified or identified later ASD in CLD children than in mainstream white children. Researchers advocate examining children for ASD within their cultural context as social cultural factors impact on ASD expression $[8,9$, 82].

A noted issue for CLD families of children with ASD is the frequent counsel of clinicians and educators that families use only one language with their affected children, and preferably the school language, as two languages are considered too challenging for these children [83, 84]. Although not much is known about the impact of bilingualism on ASD language acquisition trajectory, insistence on monolingual exposure persists, and con- tributes to challenges and distress in CLD families [83]. Research does not seem to support the belief in monolingualism for children with ASD. For example, the comparison of Ohashi et al. [85] of bilingual exposed ASD children with ASD monolinguals revealed no significant difference between groups on severity, first-word and phrase onset, or expressive-receptive-social communication scores. Hence, bilingual exposure was not disadvantageous for these young children with ASD. Similarly, a study with young bilingual English-Spanish children with ASD showed they were no different from English monolingual children with ASD in language skills, cognitive functioning, and autistic features. Bilingualism did not negatively affect language development in these young children [86]. Moreover, Hambly and Fombonne [25] found that larger vocabulary acquisition in the target or second language was best explained by larger exposure time (69\% of the variance), while expressive first language skill only explained a small portion (13\%) of the variance. They concluded that cognitive and social impairment levels are not significant variables in their second language acquisition. These findings question the wisdom in advising monolingualism for children with ASD.

\section{A Universal and Culture-Specific ASD Resource Toolkit for SLPs}

\section{Purpose of an ASD Resource Toolkit}

SLPs are not usually the first line of contact for parents concerned about their child's autism symptoms. Parents tend to first turn to their family pediatrician. These professionals are largely prepared for managing parental concerns with the availability of a resource toolkit containing information, screening, and surveillance tools, practical forms, and parent handouts to assist them in the identification, evaluation, and management of ASD [5]. Although SLPs are mostly minimally involved in the diagnostic process either because of policy or insufficient opportunities for training, it does not preclude their involvement in providing and participating in screening, surveillance, and education of stakeholders. The successful involvement of SLP with these children and their families necessitates a thorough understanding of ASD and its issues. Accordingly, a universal resource toolkit development project for SLP communities is proposed and actualized by the establishment of SLP networks within regions [72]. The toolkit is designed to increase SLP knowledge of ASD collaboratively, assist in developing culture- and language-specific tools and descriptions of 
expression of ASD in children in their communities, and serve as a valuable resource to families of children with ASD. As an example, the Autism Speaks Canada [87] 100 Day Toolkit for School-Age Children was designed to assist families of children between the ages of 5 and 13 who were recently diagnosed with autism. The site contains over 40 toolkits as well as numerous resources to support individuals with ASD and their families. Another example is the Parent Resource Kit constructed by Canada's Ontario Ministry of Children and Youth [88]. This resource was meant to act as a starting point or gateway to help parents, caregivers, and professionals find useful and credible resources on ASD. A useful resource for SLPs is ASD Pathways: a Canadian longitudinal multisite study that started in 2004 and is ongoing, as it examines predictors of positive outcomes in children with ASD age 2-11 years $[10,11]$ (findings and publications are listed on the study site).

The accessibility of a resource toolkit, modeled on that constructed for pediatric medical clinicians $[5,89]$, is meant to accomplish the following: (1) support SLPs in their efforts in caring for children with ASD by providing appropriate identification and treatment options, (2) encourage SLPs in using evidence-based gold standard early identification instruments rather than "clinical gut feeling," (3) empower SLPs in their efforts to expand their knowledge in ASD topics and advocate for a more active voice and role in all aspects of ASD needs including diagnosis [90], (4) guide SLPs in enhancing the universal resources with specific tools appropriate to their regions and the cultural and linguistic diversities within them, and (5) enable SLPs to provide education, coaching, and mentoring of families with ASD persons and other stakeholders in these children's communities.

\section{Resource Toolkit Components}

A resource toolkit is needed to enable more SLPs in becoming adept and confident in identifying and treating those with ASD. Toolkit development requires the collaborative effort of SLPs and the use of known effective resources and models. A viable resource requires both universal tools and region-culture-language-specific resources. The universal tools comprise research-based information, global resources such as websites devoted to ASD, fact sheets regarding the issues surrounding ASD, identification or observation checklists, screening tools for caregivers and educators, and parent questionnaires including narrative construction guidelines for portraying children's function in the family, cultural community, and educational setting.

Identification and Assessment of Children with Autism: A Proposed Resource Toolkit
The region-culture-language-specific resources are meant to be compiled by SLPs collaboratively with other local stakeholders, and incorporate question and answer materials addressing parental concerns and explanations of treatment types and annotated lists of local resources for all stakeholders, for example, lists and contact information of professionals well-equipped to care for children and youth with ASD $[67,69]$ such as pediatricians, psychologists, psychiatrists, and other similarly trained professionals. The lists should include dentists adept at treating children with ASD (especially those with challenging behaviors), SLPs specializing in children with ASD, occupational therapists, social services (these can provide a link to governmental funding agencies, and volunteer services such as respite resources and sitters), child care centers, and educational facilities and schools in their region. Finally, initiating a repository of narratives collected from families of children with ASD, shared by the SLP community with ASD researchers and other clinicians, can be beneficial in various ways. Such narratives would be invaluable in contributing to the better understanding of these children's needs and the challenges imposed by their ASD. It would facilitate advocating for changes in policies that hamper effective adaptation and the future of these children. The narratives would also serve the ASD research communities as they would help in tracking these children's developmental trajectories in real time. This would facilitate identifying ASD profiles, provide the impetus to the development of tools appropriate for language and culture for the identification of ASD in boys and girls in the diverse regions or populations $[79,81]$, and assist in pairing specific profiles with the "best" treatment to meet children's particular needs.

The construction of an effective resource toolkit requires that SLPs be knowledgeable about local and national governmental policies and institutional resources for families with children with ASD. Relevant documents should be available in the resource toolkit to be shared with all concerned. In addition, SLPs need to specify their ASD practice parameters. Furthermore, familiarity with screening tools suitable for training caregivers in early childhood settings [91, 92] and those used for surveillance is essential [72] so as to increase chances for early identification and accurate referral to diagnostic specialists and treatments of choice. Appendix 1 contains examples of various screening and surveillance instruments that SLPs may find useful for these purposes.

A consideration in successful involvement with families of children with ASD is sharing appropriate language of autism as it facilitates forming partnerships with them

Folia Phoniatr Logop 2017;69:27-37 DOI: $10.1159 / 000477398$ 
in the management of their children and adolescents with ASD. Kenny et al. [93] reported that while families and those with autism agreed with professionals on the terms "autism," "autism spectrum," and "autism spectrum disorder," they disagreed on "person with autism" which professionals preferred and considered as politically more correct than the term "autistic" used by a large percent of affected adults, their families, and friends. Clinicians need to be sensitive to their clients' use of terms but, at the same time, accept the language of autism used in their professional community. In addition, it is incumbent on clinicians to advise parents on language use. Families would benefit from knowledge that home language use can facilitate positive bilingual acquisition by their ASD children, and reduce parental stress that results when forced to use the school language only [83].

\section{Conclusion}

A recurring theme in the various ASD issues described here was the researchers' noted concerns about the diversity of measurement tools, methodologies, and paradigms applied in the vast numbers of studies, which hinders accurate comparisons between studies. Notwithstanding the difficulties in research, the findings highlight the depth and complexity of ASD and its manifestation in both boys and girls, and the difficulties in accurate and timely identification of this condition.

One of the issues raised here was the diversity of SLPs participation in ASD evaluation across different regions [2]. SLPs may not be directly involved in ASD evaluation; however, by becoming knowledgeable in ASD issues, and with a resource toolkit at their disposal that includes cultural and age-appropriate resources, they can be invaluable in early recognition of ASD in children and consequent appropriate referral for diagnosis. Educating parents and early child care providers in underserved populations on the importance of early identification may increase the likelihood that children with ASD receive the timely services they so obviously require and deserve [94]. Fundamental treatment goals for all children with ASD that can easily be embraced by the SLP community were best delineated by Myers and Johnson [89, p. 1162]:

The primary goals of treatment are to maximize the child's ultimate functional independence and quality of life by minimizing the core autism spectrum disorder features, facilitating development and learning, promoting socialization, reducing maladaptive behaviors, and educating and supporting families.

\section{Appendix 1}

Screening Instruments, Authors, Psychometric Utility, Detection and/or Identification of ASD and Communication Challenges

Checklist for Early Signs of Developmental Disorders: CESDD (Dereu et al., 2010 [91]; Dereu et al., 2012 [92]): screen for day care settings; author provides protocol for training CCW observation skills.

- Parents and CCWs as respondents

- Moderately high sensitivity but low specificity

- Detects ASD and delayed language development but does not differentiate between them.

Children's Communication Checklist: CCC-2 (Bishop, 2006

[71]): measure of pragmatic development.

- Parents as respondents

- High sensitivity and specificity; good PPV

- Differentiates pragmatic language disorder from speech-language impairment and detects ASD risk.

Modified Checklist for Autism in Toddlers, Revised with Follow-

Up: M-CHAT-R/F (Robins et al., 2014 [95]): a 2-stage ASD screen-

er (note: the screen is available for free download at www.m-chat. org/_references/mchatDOTorg.pdf).

- Parents as respondents

- High sensitivity and specificity; good PPV

- Best discriminating variables between ASD and other problems were measures of (1) joint attention behaviors (pointing, bringing things to show); (2) social relatedness (interest in other children, imitation); (3) communication (response to name).

Child Behavior Check List: CBCL for Toddlers (Narzisi et al., 2013 [76]): screen for ASD in 2.5- to 5-year-olds; meant mainly for pediatricians as first step (note: used successfully by an SLP, the author of this article).

- Parents as respondents

- High sensitivity, specificity, PPV, and NPV

- Only Withdrawn and Pervasive Developmental Problems subscales differentiated ASD from typical and other psychiatric disorders.

Communication and Symbolic Behavior Scales Developmental Profile Infant-Toddler Checklist: CSBS-DP (Wetherby and Prizant, 2002 in Pierce et al., 2011 [74]; Wetherby et al., 2008 [73]): a 2-stage standardized screen for communication at age 6-24 months and at chronological age 6 months to 6 years; provides profiles for speech, social, and symbolic development. Those who fail the Infant-Toddler Checklist (available in several languages (free to download at http://firstwords.fsu.edu/early.html) are also administered the Communication and Symbolic Behavior Scales.

- Parents as respondents and face-to-face evaluation by professionals (e.g., SLPs, psychologists)

- High sensitivity, specificity, and PPV

- Detects children with high ASD risk and those with ASD. Language Use Inventory: LUI (O'Neill, 2009 [96]; Pesco and O'Neill, 2012 [97]): a standardized screen for pragmatic development issues in 18- to 47-month-old English mother tongue children.

- Parents as respondents

- High sensitivity and specificity; high PPV for 24-47 months at a cutoff: $\leq 1.64 \mathrm{SD}<\mathrm{M}$

- Differentiates between typical and language-delayed children but not designed specifically for detection of ASD. 
Pragmatic Checklist (Goberis et al., 2012 [98]; Anderson, 2013 [99]): screen for pragmatic issues in the hearing-impaired (note: the screen was used successfully by the author of this article with hearing children from diverse cultures and languages to detect pragmatic challenges and plan treatment objectives).

- Parents and educators as respondents

- Provides criteria and passing scores (\%) or mastery of objectives in 6 pragmatic domains for typical children aged 24-60 months

- Detects the hearing-impaired with communication and language delays and identifies areas of weakness in their pragmatic development.
Examples of evidence-based screening instruments to be completed by parents or primary caregivers, child care workers (CCW) and educators who are well acquainted with the child, with or without assistance from professionals are shown.

ASD, autism spectrum disorder; SLPs, speech-language pathologists; PPV, positive predictive value; NPV, negative predictive value.

\section{Disclosure Statement}

No sponsorship or funding arrangements relating to this article and no conflicts of interest exist.

\section{References}

$\checkmark 1$ Weismer SE: Developmental language disorders: challenges and implications of crossgroup comparisons. Folia Phoniatr Logop 2013;65:68-77.

- Gillon G, Hyter Y, Fernandes FDM, Ferman S, Hus Y, Petinou K, Segal O, Tumanova T, Vogindroukas I, Westby C, Westerveld M: International survey of speech-language pathologists' practices in working with children with autism spectrum disorder. Folia Phoniatr Logop 2017;69:8-19.

-3 Doehring P, Volkmar FR: Knowledge gaps in ASD research: short and long term implications for policy. J Autism Dev Disord 2016;46: 733-736.

4 Volkmar F, Siegel M, Woodbury-Smith M, King B, McCracken J, State M: American Academy of Child and Adolescent Psychiatry (AACAP) Committee on Quality Issues (CQI): practice parameter for the assessment and treatment of children and adolescents with autism spectrum disorder. J Am Acad Child Adolesc Psychiatry 2014;53:237-257.

5 Johnson CP, Myers SM; Council on Children with Disabilities: Identification and evaluation of children with autism spectrum disorders. Pediatrics 2007;120:1183-1215.

6 Kirmayer LJ: Beyond the "new cross-cultural psychiatry": cultural biology, discursive psychology and the ironies of globalization. Transcult Psychiatry 2006;43:126-144.

-7 Kirmayer LJ, Rousseau C, Corin E, Groleau D: Training researchers in cultural psychiatry: the McGill-CIHR strategic training program. Acad Psychiatr 2008;32:320-326.

8 Bronfenbrenner U, Ceci SJ: Nature-nurture reconceptualized in developmental perspective: a bioecological model. Psychol Rev 1994; 101:568-586.

9 Bronfenbrenner U, Morris PA: The bioecological model of human development; in Lerner RM (ed): Handbook of Child Psychology. Hoboken, Wiley, 2006, vol 1, pp 793-828.

10 Pathways in ASD Study Team: Pathways in ASD Study: initial findings. Summaries May 2, 2011. http://www.asdpathways.ca/studyfindings/summaries/25-pathways-in-asdstudy-initial-findings.
11 Szatmari P, Georgiades S, Duku E, Bennett TA, Bryson S, Fombonne E, Mirenda P, Roberts W, Smith IM, Vaillancourt T, Volden J, Waddell C, Zwaigenbaum L, Elsabbagh M, Thompson A; Pathways in ASD Study Team: Developmental trajectories of symptom severity and adaptive functioning in an inception cohort of preschool children with autism spectrum disorder. JAMA Psychiatry 2015; 72:276-283.

12 Hatton DD, Sideris J, Skinner M, Mankowski J, Bailey DB Jr, Roberts J, Mirrett P: Autistic behavior in children with fragile $\mathrm{X}$ syndrome: prevalence, stability, and the impact of FMRP. Am J Med Genet 2006;140A:1804-1813.

13 Rice ML, Warren SF, Betz SK: Language symptoms of developmental language disorders: an overview of autism, Down syndrome, fragile $\mathrm{X}$, specific language impairment, and Williams syndrome. Appl Psycholinguist 2005;26:7-27.

14 Harris SW, Hessl D, Goodlin-Jones B, Ferranti J, Bacalman S, Barbato I, Tassone F, Hagerman PJ, Herman K, Hagerman RJ: Autism profiles of males with fragile $\mathrm{X}$ syndrome. Am J Ment Retard 2008;6:427-438.

15 Huerta M, Lord C: Diagnostic evaluation of autism spectrum disorders. Pediatr Clin North Am 2012;59:103-111.

16 Charman T, Gotham K: Measurement issues: screening and diagnostic instruments for autism spectrum disorders - lessons from research and practice. Child Adolesc Ment Health 2013;18:52-63.

17 Grandin T: My experiences as an autistic child and review of selected literature. J Orthomol Med 1984;13:144-174.

18 Grandin T: Commentary: visual abilities and sensory differences in a person with autism. Biol Psychiatry 2009;65:15-16.

19 Charman T: Developmental approaches to understanding and treating autism. Folia Phoniatr Logop 2010;62:166-177.

20 Meilleur A-AS, Fombonne E: Regression of language and non-language skills in pervasive developmental disorders. J Intellect Disabil Res 2009;53:115-124.
21 Brian AJ, Roncadin C, Duku E, Bryson SE, Smith IM, Roberts W, Szatmari P, Drmic I, Zwaigenbaum L: Emerging cognitive profiles in high-risk infants with and without autism spectrum disorder. Res Autism Spectr Disord 2014:8:1557-1566.

22 American Psychiatric Association: DSM-5, 2013. http://www.psychiatry.org/practice/ $\mathrm{dsm} / \mathrm{dsm} 5$.

23 Charman T, Pickles A, Simonoff E, Chandler $S$, Loucas T, Baird G: IQ in children with autism spectrum disorders: data from the Special Needs and Autism Project (SNAP). Psychol Med 2011;41:619-627.

24 Dromi E, Rum Y, Goldberg JF: Communication, language and speech in young children with autism spectrum disorder (ASD); in BarOn A, Ravid D (eds): Handbook of Communications Disorders: Theoretical, Empirical, and Applied Linguistic Perspectives. New York, Mouton de Gruyter, in press.

25 Hambly C, Fombonne E: Factors influencing bilingual expressive vocabulary size in children with autism spectrum disorders. Res Autism Spectr Disord 2014;8:10791089.

26 Kasari C, Brady N, Lord C, Tager-Flusberg H: Assessing the minimally verbal school-aged child with autism spectrum disorder. Autism Res 2013;6:479-493.

27 Munson J, Dawson G, Sterling L, Beauchaine T, Zhou A, Koehler E, Lord C, Rogers S, Sigman M, Estes, Abbott R: Evidence for latent classes of IQ in young children with autism spectrum disorder. Am J Ment Retard 2008; 113:439-452.

28 Charman T, Pickles A, Simonoff E, Chandler S, Loucas T, Baird G: IQ in children with autism spectrum disorders: data from the Special Needs and Autism Project (SNAP). Psychol Med 2014;41:619-627.

29 United States Environmental Protection Agency: Neurodevelopmental Disorders. America's Children and the Environment, ed 3, updated 2015. www.epa.gov/sites/production/files/2015-10/.../ace3_neurodevelopmental.pdf.
Identification and Assessment of Children with Autism: A Proposed Resource Toolkit 
30 Gillberg C: The ESSENCE in child psychiatry: early symptomatic syndromes eliciting neurodevelopmental clinical examinations. Res Dev Disabil 2010;31:1543-1551.

- 31 Lichtenstein P, Carlström E, Råstam M, Gillberg $\mathrm{C}$, Anckarsäter $\mathrm{H}$ : The genetics of autism spectrum disorders and related neuropsychiatric disorders in childhood. Am J Psychiatry 2010;167:1357-1363.

- 32 Paul R, Chawarska K, Volkmar F: Differentiating ASD from DLD in toddlers. Perspect Lang Learn Educ 2008;15:101-111.

- 33 Weismer SE, Lord C, Esler A: Early language patterns of toddlers on the autism spectrum compared to toddlers with developmental delay. J Autism Dev Disord 2010;40:1259-1273.

34 Bishop DVM: Autism and specific language impairment: categorical distinction or continuum?; in Bock G, Goode J (eds): Autism: Neural Basis and Treatment Possibilities: Novartis Foundation Symposium 251. Chichester, Wiley, 2003, vol 251, pp 213-234.

- 35 Bishop DVM: Overlaps between autism and language impairment: phenomimicry or shared etiology? Behav Genet 2010;40:618629.

- 36 Tomblin B: Co-morbidity of autism and SLI: kinds, kin and complexity. Int J Lang Commun Disord 2011;46:127-137.

- 37 Leyfer OT, Tager-Flusberg H, Dowd M, Tomblin JB, Folstein SE: Overlap between autism and specific language impairment: comparison of Autism Diagnostic Interview and Autism Diagnostic Observation Schedule scores. Autism Res 2008;1:284-296.

- 38 McGregor KK, Berns AJ, Owen AJ, Michels SA, Duff D, Bahnsen AJ, Lloyd M: Associations between syntax and the lexicon among children with or without ASD and language impairment. J Autism Dev Disord 2012;42: $35-47$.

- 39 Lindgren KA, Folstein SE, Tomblin BJ, TagerFlusberg H: Language and reading abilities of children with autism spectrum disorders and specific language impairment and their firstdegree relatives. Autism Res 2009;2:22-38.

40 Williams D, Botting N, Boucher J: Language in autism and specific language impairment: where are the links? Psychol Bull 2008;134: 944-963.

41 Tager-Flusberg H: Risk factors associated with language in autism spectrum disorder: clues to underlying mechanisms. J Speech Lang Hear Res 2016;59:143-154.

42 Klusek J, Martin GE, Losh M: Consistency between research and clinical diagnoses of autism among boys and girls with fragile $\mathrm{X}$ syndrome. J Intellect Disabil Res 2014;58:940952.

43 Baron-Cohen S: Theories of the autistic mind. Psychologist 2008;21:112-116.
44 Halladay AK, Bishop S, Constantino JN, Daniels AM, Koenig K, Palmer K, Messinger D, Pelphrey K, Sanders SJ, Tepper Singer A, Lounds Taylor J, Szatmari P: Sex and gender differences in autism spectrum disorder: summarizing evidence gaps and identifying emerging areas of priority. Mol Autism 2015; 6:36.

45 Howe Y, O’Rourke JA, Yatchmink Y, Viscidi EW, Jones RN, Morrow EM: Female autism phenotypes investigated at different levels of language and developmental abilities. J Autism Dev Disord 2015;45:3537-3549.

46 Lai MC, Lombardo MV, Auyeung B, Chakrabarti B, Simon Baron-Cohen S: Sex/gender differences and autism: setting the scene for future research. J Am Acad Child Adolesc Psychiatry 2015;54:11-24.

47 Jiménez JE, Garcia de la Cadena C, Siegel LS, O'Shanahan I, García E, Rodríguez C: Gender ratio and cognitive profiles in dyslexia: a cross-national study. Read Writ 2011;24:729747.

48 Gaub M, Carlson CL: Gender differences in ADHD: a meta-analysis and critical review. J Am Acad Child Adolesc Psychiatry 1997;36: 1036-1045.

49 May T, Cornish K, Rinehart NJ: Gender profiles of behavioral attention in children with autism spectrum disorder. J Atten Disord 2016;20:627-635

50 Auyeung B, Wheelwright S, Allison C, Atkinson M, Samarawickrema N, Baron-Cohen S: The children's empathy quotient and systemizing quotient: sex differences in typical development and in autism spectrum conditions. J Autism Dev Disord 2009;39:15091521.

51 Groen Y, Fuermaier ABM, Den Heijer AE, Tucha O, Althaus M: The empathy and systemizing quotient: the psychometric properties of the Dutch version and a review of the cross-cultural stability. J Autism Dev Disord 2015;45:2848-2864.

52 Hiller R, Young R, Weber N: Sex differences in prediagnosis concerns for children later diagnosed with autism spectrum disorder. Autism 2016;20:75-84.

53 Frazier TW, Georgiades S, Bishop SL, Hardan AY: Behavioral and cognitive characteristics of females and males with autism in the Simons Simplex Collection. J Am Acad Child Adolesc Psychiatry 2014;53:329-340.

54 Werling DM, Geschwind DH: Sex differences in autism spectrum disorders. Curr Opin Neurol 2013;26:146-153.

55 Baron-Cohen S, Cassidy S, Auyeung B, Allison C, Achoukhi M, Robertson S, Pohl A, Laiet MC: Attenuation of typical sex differences in 800 adults with autism vs. 3,900 controls. PLoS One 2014;9:e102251.

56 Gould J, Ashton-Smith J: Missed diagnosis or misdiagnosis? Girls and women on the autism spectrum. GAP 2011;12:34-41.

57 Szalavitz M: The invisible girls. Sci Am Mind 2016;27:48-55.
58 Rosin S: Practical tips to differentiate social communication disorder from autism spectrum disorder; Streaming Video \& PDF, ASHA Case Studies Course 15255, October 4, 2015. http://www.asha.org/Professional-Development/case-studies/.

59 Esbensen AJ, Mailick Seltzer M, Lam KSL, Bodfish JW: Age-related differences in restricted repetitive behaviors in autism spectrum disorders. J Autism Dev Disord 2009;39: $57-66$.

60 Grossman R: The ASD Assessment Scale/ Screening Questionnaire (based on DSM5 Criteria). Childbrain.com, 2014: The Pediatric Neurology Site. http://www.childbrain. com/pddq6.shtml.

61 Norbury CF: Practitioner review: social (pragmatic) communication disorder conceptualization, evidence and clinical implications. J Child Psychol Psychiatry 2014;55: 204-216.

62 Brukner-Wertman Y, Laor N, Golan O: Social (pragmatic) communication disorder and its relation to the autism spectrum: dilemmas arising from the DSM-5 classification. J Autism Dev Disord 2016;46:2821-2829.

63 Lalkhen AG, McCluskey A: Clinical tests: sensitivity and specificity. $\mathrm{Br} \mathrm{J}$ Anaesth CEACCP 2008;8:221-223.

64 McGee S: Simplifying likelihood ratios. J Gen Intern Med 2002;17:647-650.

65 Lord C, Rutter M, Le Couteur A: Autism Diagnostic Interview-Revised: a revised version of a diagnostic interview for caregivers of individuals with possible pervasive developmental disorders. J Autism Dev Disord 1994; 24:659-685.

66 Lord C, Rutter M, DiLavore PC, Risi S, Gotham K, Bishop S: Autism diagnostic observation schedule: ADOS-2. Los Angeles, Western Psychological Services, 2012.

67 Falkmer T, Anderson K, Falkmer M, Horlin $\mathrm{C}$ : Diagnostic procedures in autism spectrum disorders: a systematic literature review. Eur Child Adolesc Psychiatry 2013;22:329-340.

68 Gray MK, Tonge BJ, Sweeney DJ: Using the Autism Diagnostic Interview-Revised and the Autism Diagnostic Observation Schedule with young children with developmental delay: evaluating diagnostic validity. J Autism Dev Disord 2008;38:657-667.

69 Kamp-Becker I, Ghahreman M, HeinzelGutenbrunner M, Peters M, Remschmidt H, Becker K: Evaluation of the revised algorithm of Autism Diagnostic Observation Schedule (ADOS) in the diagnostic investigation of high-functioning children and adolescents with autism spectrum disorders. Autism 2013;17:87-102.

70 Lord C: What can we learn about treatment and intervention for ASD from a longitudinal study from 2-22? Gold-standard diagnosis in diverse and multicultural settings [PDF]. Donald Cohen Research, Practice and Policy Series Workshop April 7, 2015, McGill University, Montreal, Canada. 
71 Bishop DVM: CCC-2: Children's Communication Checklist-2 United States Edition Manual. San Antonio, NCS Pearson, 2006.

72 Robins DL: Screening for autism spectrum disorders in primary care settings. Autism 2008; $12: 537-556$

73 Wetherby AM, Brosnan-Maddox S, Peace V, Newton L: Validation of the Infant-Toddler Checklist as a broadband screener for autism spectrum disorders from 9 to 24 months of age. Autism 2008;12:487-511.

74 Pierce K, Carter C, Weinfeld M, Desmond J, Hazin R, Bjork R, Gallagher N: Detecting, studying, and treating autism early: the oneyear well-baby check-up approach. J Pediatr 2011;159:458-465.

-75 Garcia-Primo P, Hellendoorn A, Charman T, Roeyers H, Dereu M, Roge B, Baduel S, Muratori F, Narzisi A, Van Daalen E, Moilanen I, Posada de la Paz M, Canal-Bedia R: Screening for autism spectrum disorders: state of the art in Europe. Eur Child Adolesc Psychiatry 2014;23:1005-1021.

-76 Narzisi A, Calderoni S, Maestro S, Calugi S, Mottes E, Muratori F: Child Behavior Check List $1 \frac{1 / 2}{2}-5$ as a tool to identify toddlers with autism spectrum disorders: a case-control study. Res Dev Disabil 2013;34:1179-1189.

-77 Siu AL; US Preventive Services Task Force (USPSTF): Screening for autism spectrum disorder in young children US Preventive Services Task Force recommendation statement. JAMA 2016;315:691-696.

78 Barton EE, Harris B, Leech N: Autism identification policies and practices in early childhood: a preliminary investigation from one state. Infants Young Child 2016;29:267-289.

79 Daley TC: The need for cross-cultural research on the pervasive developmental disorders. Transcult Psychiatry 2002;39:531-550.

80 Hussein H, Taha Ghada RA, Almanasef A: Characteristics of autism spectrum disorders in a sample of Egyptian and Saudi patients: transcultural cross sectional study. Child Adolesc Psychiatry Ment Health 2011;5:34.
81 Zuckerman KE, Mattox K, Donelan K, Batbayar O, Baghaee A, Bethell C: Pediatrician identification of Latino children at risk for autism spectrum disorder. Pediatrics 2013;132: 445-451.

82 Kirkovski M, Enticott PG, Fitzgerald PB: A review of the role of female gender in autism spectrum disorders. J Autism Dev Disord 2013;43:2584-2603.

83 Park S: Bilingualism and children with autism spectrum disorders: issues, research, and implications. NYS TESOL J 2014;1:122-129.

84 Yu B: Issues in bilingualism and heritage language maintenance: perspectives of minoritylanguage mothers of children with autism spectrum disorders. Am J Speech Lang Pathol 2013;10:10-24.

85 Ohashi JK, Mirenda P, Marinova-Todd S, Hambly C, Fombonne E, Szatmari P, Bryson S, Roberts W, Smith I, Vaillancourt T, Volden J, Waddell C, Zwaigenbaum L, Georgiades S, Duku E, Thompson A; Pathways in ASD Study Team: Comparing early language development in monolingual- and bilingual-exposed young child with autism spectrum disorders. Res Autism Spectr Disord 2012;6: 890-897.

86 Valicenti-McDermott M, Tarshis N, Schouls M, Galdston M, Hottinger K, Seijo R, Shulman L, Shinnar S: Language differences between monolingual English and bilingual English-Spanish young children with autism spectrum disorders. J Child Neurol 2012;28: 945-948.

87 Autism Speaks Canada: Toolkits, 2017. http:// www.autismspeaks.ca/science-and-services/ resources/tool-kits/.

88 Ontario Ministry of Children and Youth: Autism parent resource kit, 2017. http://www. children.gov.on.ca/htdocs/English/specialneeds/autism/aprk/index.aspx.

89 Myers SM, Johnson CP: Management of children with autism spectrum disorders. Pediatrics 2007; 120:1162-1182.

90 McClure I, Mackay T, Mamdani H, McCaughey R: A comparison of a specialist autism spectrum disorder assessment team with local assessment teams. Autism 2010;14:589603.
91 Dereu M, Warreyn P, Raymaekers R, Meirsschaut M, Pattyn G, Schietecatte I, Roeyers H: Screening for autism spectrum disorders in Flemish day-care centres with the Checklist for Early Signs of Developmental Disorders. J Autism Dev Disord 2010;40:1247-1258.

92 Dereu M, Raymaekers R, Warreyn P, Schietecatte I, Meirsschaut M, Roeyers H: Can child care workers contribute to the early detection of autism spectrum disorders? A comparison between screening instruments with child care workers versus parents as informants. J Autism Dev Disord 2012;42:781-796.

93 Kenny L, Hattersley C, Molins B, Buckley C, Povey C, Pellicano E: Which terms should be used to describe autism? Perspectives from the UK autism community. Autism 2015;1: $1-21$.

94 Daniels AM, Mandell DS: Explaining differences in age at autism spectrum disorder diagnosis: a critical review. Autism 2014;18: 583-597.

95 Robins DL, Casagrande K, Barton ML, Chen CM, Dumont-Mathieu T, Fein D: Validation of the modified checklist for autism in toddlers, revised with follow-up (M-CHATR/F). Pediatrics 2014;133:37-45.

96 O'Neill DK: Language Use Inventory - LUI Manual 2009. Waterloo, Knowledge in Development, 2009.

-97 Pesco D, O’Neill DK: Predicting later language outcomes from the Language Use Inventory. J Speech Lang Hear Res 2012;55: 421-434.

98 Goberis D, Beams D, Dalpes M, Abrisch-Baca A, Yoshinaga-Itano C: The missing link in language development of deaf and hard of hearing children: pragmatic language development. Semin Speech Lang 2012;33:297309.

99 Anderson KL: Social Communication Skills: The Pragmatic Checklist. Supporting Success for Children with Hearing Loss, 2013. https:// successforkidswithhearingloss.com.
Identification and Assessment of Children with Autism: A Proposed Resource Toolkit
Folia Phoniatr Logop 2017;69:27-37 